\title{
Electrophysiological correlates of high-level perception during spatial navigation
}

\author{
Christoph T. Weidemann, Matthew V. Mollison, and Michael J. Kahana \\ University of Pennsylvania, Philadelphia, Pennsylvania
}

\begin{abstract}
We studied the electrophysiological basis of object recognition by recording scalp electroencephalograms while participants played a virtual-reality taxi driver game. Participants searched for passengers and stores during virtual navigation in simulated towns. We compared oscillatory brain activity in response to store views that were targets or nontargets (during store search) or neutral (during passenger search). Even though store category was solely defined by task context (rather than by sensory cues), frontal electrophysiological activity in the low frequency bands (primarily in the $\theta[4-8 \mathrm{~Hz}]$ band) reliably distinguished between the target, nontarget, and neutral store views. These results implicate low-frequency oscillatory brain activity in frontal regions as an important variable in the study of the cognitive processes involved in object recognition, categorization, and other forms of high-level perception.
\end{abstract}

Humans and other animals use locomotion to accomplish various goals, such as finding shelter or food. For navigation in the environment to be successful, sensory input has to be mapped to high-level percepts that can range from very general (e.g., "shelter") to very specific (e.g., "my home"). The current goals help to flexibly shape these percepts to allow us, for example, to view a bench, a tree stump, and a low wall as all belonging to the goal category when searching for an object to sit on, yet to recognize only one of many chairs as the goal object when returning to a seat in a restaurant. In this article, we investigate the neurophysiological basis of this fast and effortless process of high-level perception in the context of spatial navigation.

Similar perception and decision processes are often studied in laboratory tasks that require no locomotion. In visual search, for example, the sensory input for a display is mapped to high-level "target" and "nontarget" percepts on which the response is based (see, e.g., Wolfe, 1998, for a review). Just as certain landmarks may be the goal of spatial search in some cases and not in others, the same stimuli can act as targets in some trials and nontargets in others during visual search. These studies have contributed significantly to our understanding of how visual features are integrated into percepts and of the role of attention in the parsing of visual scenes.

However, the simultaneous presentation of all stimuli that is typical in visual search studies makes it difficult, if not impossible, to investigate the processes that give rise to the perception of any particular stimulus within this display and to its classification as a target or nontarget. Better suited for this purpose are studies that show one stimulus at a time in a rapid serial visual presentation (RSVP). In some RSVP studies (especially those examining repetition blindness-e.g., Kanwisher, 1987), every stimulus is effectively a target, in the sense that each stimulus has to be kept in memory until all stimuli have been presented. In other studies (especially those examining the attentional blink-e.g., Raymond, Shapiro, \& Arnell, 1992), only a small subset of the stimuli are targets, making the studies more conceptually similar to studies of visual search (see Chun, 1997, for a review of these types of RSVP studies). RSVP studies, as well as other studies presenting multiple stimuli nearby in time and space, have consistently shown that the stimuli presented close to a target profoundly affect its processing (e.g., Huber, Shiffrin, Lyle, \& Ruys, 2001; Meyer \& Schvaneveldt, 1971, 1976; Weidemann, Huber, \& Shiffrin, 2005, 2008). Such studies provide important insights into the processes involved in integrating and differentiating sensory input into distinct percepts. This influence of nearby stimuli on the processing of the target, however, makes it difficult to study the formation of high-level percepts separately from the processes involved in parsing the sensory input.

Naturally, the issue of effects from nearby stimuli contaminating the study of target processing is not limited to the behavioral data discussed above, but applies at least as much to neurophysiological recordings, even when these provide high temporal resolution. Oscillations in electroencephalogram (EEG) activity have frequently been tied to task variables and cognitive processes. These effects manifest themselves at a wide range of oscillatory frequencies, including low frequency bands in the $\delta(2-4 \mathrm{~Hz})$ and especially the $\theta(4-8 \mathrm{~Hz}$ in humans, $4-12 \mathrm{~Hz}$ in rats) ranges (e.g., Hwang et al., 2005; Jacobs, Hwang, Curran, \& Kahana, 2006; Sederberg et al., 2006; see Kahana, 2006, for a review). Indeed, a common form of analysis is to average time-locked trials to study event-related potentials (ERPs), which tend to emphasize low-frequency fluctuations in recorded EEG activity. Reliable ERP components have been identified several hundred milliseconds after the offset of

C.T.Weidemann, ctw@cogsci.info 
the event to which they correspond (e.g., a stimulus presentation). To avoid a superimposition of many similar effects, these components are usually studied by introducing temporal separations on the order of seconds between stimulus presentations. In RSVP tasks, stimuli are presented at a much higher rate, which makes it very difficult to study the effects of particular stimulus presentations in the RSVP sequence (Vogel, Luck, \& Shapiro, 1998).

When navigating through the world, salient landmarks can be separated arbitrarily, which facilitates the study of their recognition. Moreover, sensory input during navigation in natural environments provides other important advantages: As in visual search tasks, the target object is presented within a context of irrelevant stimuli, but this context often shares few features with the target, making the target easier to identify. Furthermore, spatial search in natural settings often imposes strong constraints on possible target locations, which can severely reduce the need to engage in visual search (e.g., a quick glance at an intersection can be enough to determine whether or not a traffic light is present). Studying the neurophysiological correlates of spatial search before and after onsets of the target and of similar nontarget stimuli can therefore provide important insights into the neural basis of highlevel perception that are difficult to obtain from more traditional experimental paradigms.

\section{High-Level Perception During Spatial Navigation}

To present target stimuli sufficiently far apart to allow for reliable estimates of low-frequency fluctuations in the EEG signal while still keeping participants engaged, we asked participants to play the role of a taxi driver in an immersive virtual-reality computer game. In this game, participants navigated in a virtual town with specific goals that varied from trial to trial, so that a particular store could be the goal location on one trial and irrelevant on the next. Although this dynamic and interactive display afforded less control over the timing of stimulus presentations than do more traditional experimental paradigms, it allowed us to separate target and nontarget objects in (virtual) space so that the separation between subsequent objects was on the order of seconds. The interactive nature of this task had the advantage of keeping participants engaged continuously, despite the large temporal separations between presentations of potentially relevant objects.

\section{METHOD}

\section{Participants}

Twenty adults (ages 19-29 years, $M=22 ; 11$ male, 9 female) participated in three experimental sessions for monetary compensation $(\$ 15 / \mathrm{h}$, plus a performance-based bonus of up to $\$ 10 /$ session). All participants were right-handed native English speakers and had normal or corrected-to-normal vision. Each session, including application of the electrode net and running in the task, lasted approximately $90 \mathrm{~min}$.

\section{EEG Recordings}

A 128-channel geodesic sensor net was used to measure EEGs at the scalp with a sampling rate of $500 \mathrm{~Hz}$. The net was connected to an AC-coupled, high-input-impedance amplifier (200 M 2 ; Net
Amps, Electrical Geodesics Inc., Eugene, OR). The electrodes were adjusted until the impedances were less than $50 \mathrm{k} \Omega$.

A central vertex reference $(\mathrm{Cz})$ was used during recording, and all analyses were based on referencing to the average of all electrodes (Dien, 1998). Channels deemed too noisy by visual inspection were excluded from the average during the referencing process, as well as from all subsequent analyses. Furthermore, events with eye-movement or eye-blink artifacts (i.e., those with an electrooculogram exceeding $\pm 100 \mu \mathrm{V}$ ), as well as those in which the measured voltage fell outside the range of $\pm 75 \mu \mathrm{V}$, were also excluded from the analyses. To simplify the analyses and increase their power to detect regional differences, we grouped the electrodes into eight regions of interest (ROIs) inspired by those used in other studies (e.g., Curran, 2004; Curran, DeBuse, \& Leynes, 2007; Curran, DeBuse, Woroch, \& Hirshman, 2006; Curran \& Friedman, 2004; Curran \& Hancock, 2007). The shaded regions in Figure 1 illustrate these ROIs, and only data from electrodes in these ROIs were analyzed.

\section{Procedure}

Participants played the role of a taxi driver within a virtual, 3-D town. In each trial, they were first required to navigate through the town looking for passengers (passenger search). Once a passenger was picked up, the participant was instructed to drive him or her to a particular store within the town (store search). Participants were urged to find the target locations as quickly and accurately as possible by using the fastest route. Each experimental session consisted of three test towns, with 15 passenger deliveries per town. The first and second towns in each session were novel, and the third was identical to the first. ${ }^{1}$ Each town was laid out on a $6 \times 6$ grid of blocks (see Figure 2A), with a single store or background building on each block. Five stores and 31 background buildings were randomly placed in each town, subject to the requirement that stores could not be placed in adjacent locations or in all four corners of the town.

At the beginning of the experiment, the participants first delivered passengers to stores in a small $(3 \times 3$ block $)$ practice town to familiarize them with navigation in the virtual environment (these stores were not reused in the three larger test towns). Before entering each of the test towns, participants viewed static 2-D images of all 10 storefronts with the store names to ensure that they were highly familiar with the appearance of the stores (this list was presented five times, each time in a new random order, and participants were asked to passively view it to familiarize themselves with the stores).$^{2}$

Participants navigated from a first-person perspective using a computer-game controller, which was held with both hands resting in the lap, thereby reducing arm movements that could cause muscle artifacts in the electrophysiological recordings. ${ }^{3}$ For the first delivery in every town, a passenger was placed directly in front of the virtual taxi. During each of the remaining passenger search phases, six passengers were distributed at different random locations within the town, such that at most one passenger was on any given block and a passenger usually was not in the line of sight of the upcoming target store. Figure $2 \mathrm{~B}$ shows a typical view during a passenger search phase. Participants were asked to visit all five stores before a store was requested again. To motivate participants to learn the layout of each town, the monetary bonus was based on how fast the deliveries were made.

\section{Data Analyses}

The dynamic and interactive nature of the display presented a challenge when trying to time-lock the analyses to the presentation of (potential) target stimuli. When the stimuli first appeared on-screen, they were typically viewed from a distance, only becoming discernible as they were approached. All of our analyses concerned the processing of store stimuli, and we time-locked the analyses to the moment in which a given store presentation exceeded a minimum size threshold ( $0.35 \%$ of the display). We chose this threshold on the basis of preliminary analyses that indicated that target stores were directly approached on $95 \%$ of trials in which this threshold was exceeded. Figure $2 \mathrm{C}$ illustrates a typical view of a store at that threshold. 


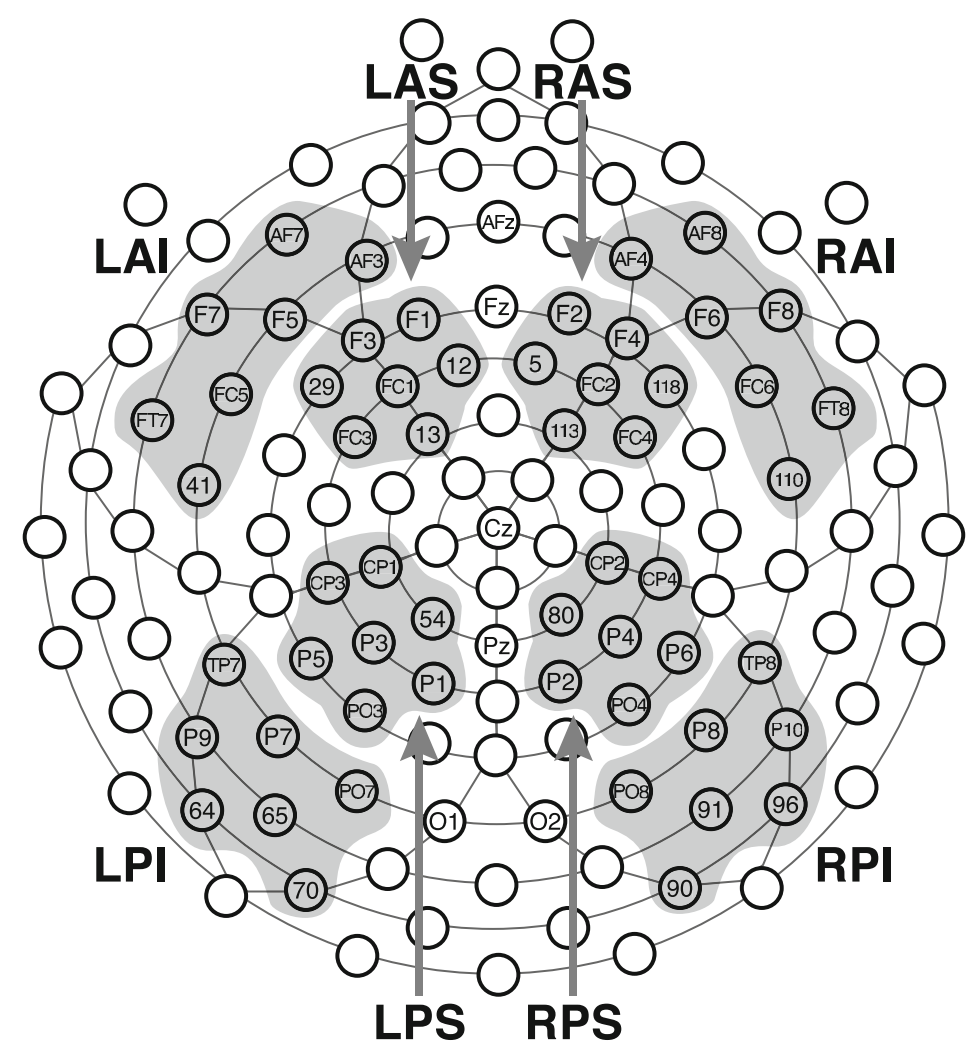

Figure 1. The 128-channel geodesic sensor net used to measure the EEGs and regions of interest (ROIs) on which the analyses were based. Each ROI is labeled with a three-letter name that describes its position on the skull: $R=$ right, $L=$ left, $A=$ anterior, $P=$ posterior, $S=$ superior, $I=$ inferior.

We classified stores as targets and nontargets during store search, and as neutral during passenger search. We limited the analyses to events in which only a single store was viewed and stayed in view for at least $1.5 \mathrm{sec}$ after it surpassed the size threshold described above. We only considered target store events in which the participant arrived at the store without it leaving the field of view, and we only considered neutral store events in which no passenger was visible (a passenger was only considered to be present if it occupied more than $0.35 \%$ of the screen at any time during the event). In total, we identified 2,421,2,068, and 1,725 target, nontarget, and neutral events, respectively, but a substantial proportion of these events was discarded because of eye-movement artifacts, leaving 1,225, 1,494, and 1,014 target, nontarget, and neutral events, respectively. ${ }^{4}$ The median time intervals between successive events of the same kind (again, considering only artifact-free events) were 62, 55, and $63 \mathrm{sec}$ for target, nontarget, and neutral events, respectively; the median time between any two artifact-free events was $23 \mathrm{sec}$.

To minimize potential issues arising from variability in store detection relative to the threshold described above, and to investigate high as well as low oscillatory frequencies in EEG activity, we calculated the oscillatory power for different frequencies at each trial and electrode and averaged only measures derived from this oscillatory power, rather than averaging raw voltages, as is done in ERP analyses. We calculated oscillatory power using Morlet wavelets with a width of 5 , for 23 logarithmically spaced frequencies between 2 and $90.5 \mathrm{~Hz}$ $\left(\left\{2^{x}\right\}\right.$, where $x \in\{1,1.25,1.5, \ldots, 6.5\}$; cf. Jensen \& Tesche, 2002;
A

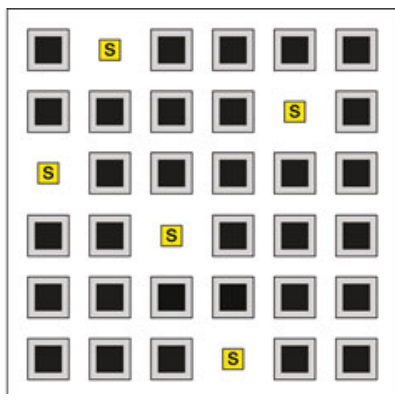

B

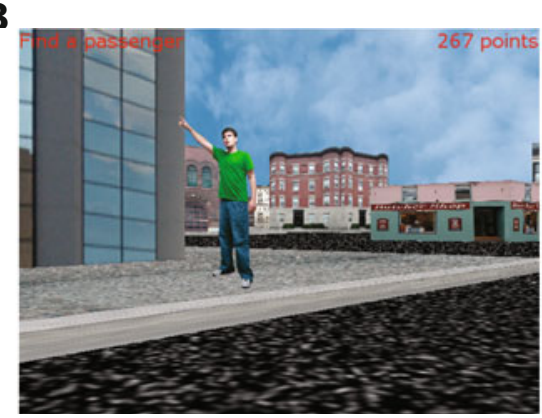

C

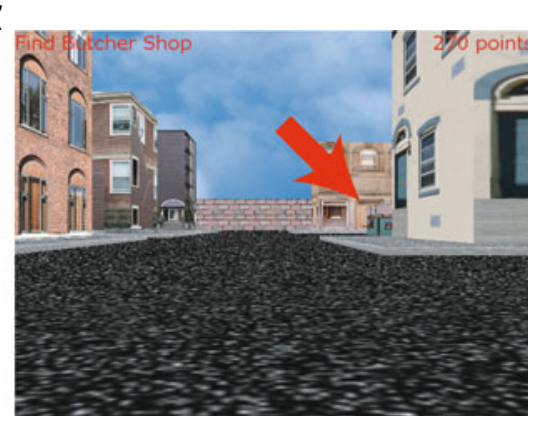

Figure 2. (A) Top-down view of a possible town layout. Squares with an "s" inside indicate store locations; dark squares surrounded by a lighter area indicate building and sidewalk locations. (B) A passenger, store, and buildings within the virtual town. (C) The store in this figure occupies approximately $0.35 \%$ of the display (arrow added for illustrative purposes). 
Tallon-Baudry, Bertrand, Delpuech, \& Permier, 1997). In contrast to other electrophysiological measures, such as phase-locking values and ERPs, this procedure is relatively robust to variations in the true detection times, as measured with respect to the threshold defined above.

\section{RESULTS}

As in previous studies investigating spatial navigation in simple virtual towns, in which passenger search phases allowed for ample exploration (Newman et al., 2007), participants learned the layouts of the towns quickly and took efficient routes to the target stores. (For each town, the majority of deliveries consisted of routes that exceeded the city-block distance between the passenger pick-up location and the target store by less than one unit.) Because navigation performance was so high across towns, and because our focus is on the perception of target, nontarget, and neutral stimuli rather than on spatial navigation or learning, we have aggregated across towns and deliveries in all subsequent analyses. Detailed behavioral analyses of spatial navigation and learning in a task very similar to the one used here are reported by Newman et al.

To investigate how oscillations in EEG activity covary with high-level perception, we compared oscillatory activity after the onset of target, nontarget, and neutral stores with activity for a baseline period before store onset. As detailed below, we found strong and widespread effects in response to all stores in the lower frequency bands that differentiated between the high-level store categories in the frontal ROIs.

Specifically, we aggregated oscillatory power (for each participant in each session) over ROIs (see Figure 1) and over time windows of $0.3 \mathrm{sec}$, starting $0.6 \mathrm{sec}$ before the onset of the store (as defined by the threshold previously described) and until $1.5 \mathrm{sec}$ after store onset. The first time window (0.6-0.3 sec before store onset) served as a baseline, and power in all time windows was $z$-transformed by subtracting the mean baseline power and dividing by the standard deviation of the baseline power. We chose this baseline because the associated 300-msec buffer between its end and the store onset allowed us to reasonably assume that it mainly indexed electrophysiological activity prior to the detection of the store. Thus, the transformed values index the standardized change in power associated with the detection of a store.

We then aggregated the data into frequency bands by averaging over frequencies in the $\delta(2-4 \mathrm{~Hz}), \theta(4-8 \mathrm{~Hz})$, $\alpha(8-16 \mathrm{~Hz}), \beta(16-32 \mathrm{~Hz})$, and $\gamma(\geq 32 \mathrm{~Hz})$ ranges. Figure 3 shows the $z$-transformed power values for target, nontarget, and neutral events in the different frequency bands and ROIs as a function of time.

As is evident from the figure, oscillatory power in the lower frequencies decreased around the onset of a store. Aggregating across store categories, ROIs, and time windows after store onset confirmed significantly negative $z$-transformed power for the $\delta, \theta$, and $\alpha$ frequency bands $[t(19)=-9.14,-8.78$, and -3.12 , respectively; $S E=$ $0.03,0.02$, and $0.03 ; p<.01$ for all comparisons]. Furthermore, oscillatory power in the anterior ROIs exhibited a tendency to diverge for target, nontarget, and neu- tral store views. To determine whether the power values for these different event types differed significantly, we performed three permutation tests, one for each possible pairwise comparison. In analogy to $t$ tests, these permutation tests were based on the relative differences in $z$-transformed power between two event types across participants. To compare target and nontarget events, for example, we subtracted the mean nontarget power for each participant at each ROI and time window from the corresponding mean target power and then averaged this difference across participants. We then divided this mean difference in power for each ROI and time window by its standard error to get the relative differences in power between the two conditions.

We compared each relative difference in power to the distribution of relative differences obtained by permuting the condition labels at the level of participants. For 20 participants, there are $2^{20}$ possible ways to permute the labels for two conditions, but because inverting all labels just changes the sign of the relative difference, only half of these need to be considered. To illustrate this process, consider comparing data for target and nontarget events: Each combination of frequency band, ROI, and time window yields a relative difference between these two conditions. A permutation distribution can be obtained by flipping the two labels for subsets of the participants, such that for some participants the data from target events are treated as data from nontarget events, and vice versa (as described above, there are $2^{20}$ different ways to do this). If $z$-transformed power does not distinguish between target and nontarget events, we would expect the observed relative difference of power between these two conditions to be close to the center of the distribution of relative differences obtained from these permutations. We compared the observed absolute relative differences in power with the distribution of absolute relative differences on the basis of these permutations and deemed a difference to be statistically significant if it exceeded the 95 th percentile of this distribution. By using only one overall significance criterion per test, the family-wise Type I error rate for each of the three comparisons was fixed at $5 \%$ across all frequency bands, ROIs, and time windows (cf. Maris \& Oostenveld, 2007; Nichols \& Holmes, 2002).

Figure 3 shows significant differences in oscillatory power between neutral and target stores that were prevalent in the left and right anterior inferior ROIs for the $\delta$, $\theta$, and $\alpha$ frequency bands; this difference can also be observed in the left and right anterior superior ROI for the $\theta$ frequency band and was always such that oscillatory power was lower for target than for neutral stores. In the anterior inferior ROIs (with the exception of the left anterior inferior ROI), there are also significant differences between oscillatory power for target and nontarget events at the three lowest frequency bands, in which oscillatory power was lower for target stores than for nontarget stores. Furthermore, the right anterior superior ROI showed significantly lower oscillatory power for nontarget than for neutral events directly after store onset (all other effects only reached significance at least $0.3 \mathrm{sec}$ after store onset). Because differences in low-frequency oscillations in EEG 

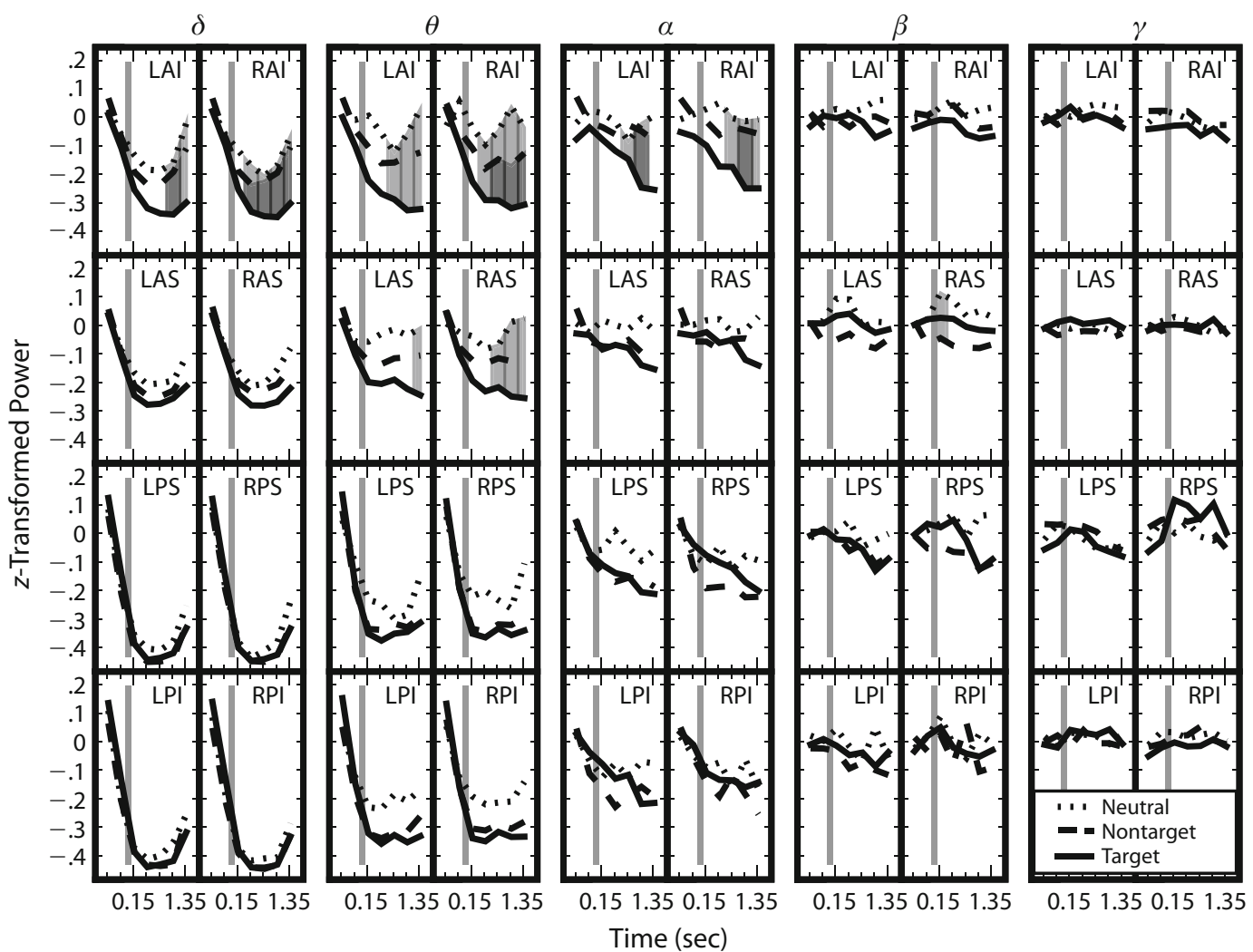

Figure 3. Plots of $z$-transformed power for the neutral, nontarget, and target store views. The labels on the abscissa show the mean time point of each bin (data from the first two time points in each panel, separated by a gray vertical line, are from before the store presentation exceeded threshold). Because of the $z$ transformation, the means in each panel are constrained to start near zero (because of different numbers of target, nontarget, and neutral observations, the central tendency of these means can deviate slightly from zero). The five columns show $z$-transformed power for the $\delta(2-4 \mathrm{~Hz}), \theta(4-8 \mathrm{~Hz}), \alpha(8-16 \mathrm{~Hz}), \beta(16-32 \mathrm{~Hz})$, and $\gamma(\geq 32 \mathrm{~Hz})$ frequency bands. From top to bottom, the panels show data from the left and right anterior inferior (LAI, RAI), anterior superior (LAS, RAS), posterior superior (LPS, RPS), and posterior inferior (LPI, RPI) regions of interest. The shaded gray areas show significant differences. Except for one panel in the $\beta$ band, which shows a significant difference between neutral and nontarget store views in the RAS region, light gray areas show significant differences between neutral and target views, whereas dark gray areas denote significant differences between nontarget and target views.

activity (most notably in the $\theta$ band) have been linked to differences in (virtual) movement (see, e.g., Caplan et al., 2003; Ekstrom et al., 2005), we repeated our analyses using only a subset of events in which participants moved continuously from the beginning of the baseline period until $1.5 \mathrm{sec}$ after store onset. Despite being considerably more noisy because of the reduced number of data points, these results showed the same qualitative pattern as the full data set.

\section{DISCUSSION}

We investigated oscillations in EEG activity relative to the onset of stimuli that, on some trials, were the targets of spatial search during navigation in a virtual environment. Our analysis focused on comparing electrophysiological activity in response to goal stimuli (target stores) with that for the same stimuli on trials in which either a stimulus from the same category was the target (i.e., nontarget stores, when a store was the target) or when participants searched for categorically different stimuli (i.e., neutral stores, when participants were searching for passengers). We observed a widespread decrease in low-frequency oscillatory power around the time of store onset for all store types. This decrease was especially pronounced for target stimuli, in that frontal oscillatory power was significantly lower for these than for neutral stimuli, predominantly in the $\theta$ range but also extending to neighboring frequency ranges (see Figure 3). Differences between target and nontarget stimuli showed a similar pattern of results (albeit with smaller effect size), but, with the exception of one time point at one ROI, the differences between neutral and nontarget stimuli failed to reach significance. Strikingly, all significant differences between the conditions were confined to the frontal ROIs.

These results extend those of Caplan et al. (2003), who observed greater $\theta$ power for passenger search than for store search in a similar taxi driver game without conditioning on store view. Even though the natural and interactive nature of our task makes it impossible to completely rule out behavioral confounds that may have contributed to these results (such as potential differences in small 
eye movements across conditions and/or in response to all stores), note that we would expect different levels of microsaccades to produce artifactual results in the $\gamma$ band (Yuval-Greenberg, Tomer, Keren, Nelken, \& Deouell, 2008), where we found no differences between conditions and no difference in oscillatory power from baseline. Also note that our results are characterized by widespread and robust low-frequency desynchronizations that vary in strength across stimulus classes at frontal ROIs - a pattern unlikely to be due to muscle artifacts, especially given that we matched the different event types as closely as possible in terms of their perceptual and navigational characteristics. The fact that the same stimuli served as target, nontarget, and neutral stores suggests that any differences between conditions likely result from top-down processes that match stores to the current goal.

Another possible concern is that different cognitive strategies could potentially be used between the passenger search and store search phases of the experiment. Such differences would likely have affected nontarget store views (during store search phases) and neutral store views (during passenger search phases) differently, yet the differences between these conditions were (with one exception) small and not statistically significant. Our analyses of relative change with respect to baseline periods before store onset likely substantially reduced or eliminated the potential effects of different cognitive strategies, and therefore these results are not well suited to evaluate the global effects of different strategies in spatial search.

It is particularly interesting that oscillatory brain activity reliably distinguished between visual stimuli on the basis of only their trial-specific roles. To perceive a given stimulus as "target" on some trials and not on others requires the integration of ongoing sensory input with the current goals and knowledge. It is tempting to conclude from our results that widespread cortical networks that (de)synchronize at low frequencies directly index processes that are sensitive to the detection of salient stimuli with frontal networks specializing in the formation of high-level percepts based on the current goals. The special role of the frontal ROIs seems compatible with several imaging studies that have particularly implicated frontal brain regions in visual categorization (see, e.g., Vogels, Sary, Dupont, \& Orban, 2002, for a review). This interpretation suggests that decreases in low-frequency oscillatory power may also be able to index target detection in other tasks in which targets are not well separated in time and/or space from other salient stimuli, such as visual search or RSVP tasks. This would make low-frequency oscillatory power an important dependent variable to consider when studying target detection, even when the task is not well suited for conventional stimulus-locked EEG analyses.

An equally plausible explanation for our results, however, is that the observed differences index associated processes that depend on the formation of high-level percepts (rather than directly indexing the processes responsible for forming such percepts). Such secondary processes could include those associated with orienting and route planning: Even though the participants learned the environment well in our study, it is reasonable to assume that the relative routes between stores were especially well encoded so that viewing a store decreased uncertainty about the location within the environment and about which way to proceed. To the extent that low-frequency oscillatory activity reflects ongoing orienting and route-planning processes, viewing a store should decrease the demand of those processes. This could explain the widespread decrease in low-frequency oscillatory power for all store types, as well as the stronger decrease for target stores in frontal ROIs (because target stores signal the goal of the ongoing navigation). Likewise, the decrease in demand of these processes should be least when all stores are neutral with respect to target status, just as we observed. This interpretation of our results is consistent with studies showing the recruitment of frontal brain areas for route learning (e.g., Shelton \& Gabrieli, 2002) and single-unit studies highlighting the importance of frontal regions in goal-directed navigation (e.g., Hok, Save, LenckSantini, \& Poucet, 2005; Poucet et al., 2004).

Alternatively, our results may reflect attentional and/or working memory effects: While searching for a particular store, encountering this goal is likely associated with a release of working memory (i.e., the current goal does not have to be actively maintained anymore), and attention likely remains focused on the target store. Encountering a nontarget store, on the other hand, only helps narrow down the target search, and attention likely shifts away from that stimulus as it is passed. Similarly, neutral stores are of little relevance during passenger search, and we would therefore expect any attentional or working memory effects to be smallest for these stimuli. This interpretation of our results is consistent with several studies relating $\theta$ oscillations to working memory and task demands (e.g., Jensen \& Tesche, 2002; Sauseng, Hoppe, Klimesch, Gerloff, \& Hummel, 2007). The particular sensitivity of frontal regions to attentional effects seems compatible with a recent imaging study assessing the processing of unexpected events during spatial navigation in humans (Iaria, Fox, Chen, Petrides, \& Barton, 2008).

If our results index secondary processes that depend on the formation of high-level percepts, the extent to which the findings generalize to different tasks, such as visual search and RSVP tasks, would depend on the specific nature of these processes. Attentional processes may produce similar results even for superficially very different tasks, whereas other processes, such as those required for orienting and route planning, would be more specific to tasks involving spatial navigation.

Future work will be required in order to characterize whether the measures we have used assess the formation of high-level percepts directly or indirectly. Regardless of the ultimate source of our effects, their clear correspondence with high-level percepts can establish strong constraints for theories addressing the formation and time course of these effects and, in turn, for theories of decision making, categorization, and other aspects of human cognition.

\section{AUTHOR NOTE}

M.V.M. is now at the Department of Psychology at the University of Colorado at Boulder. The authors acknowledge Igor Korolev's help with the data acquisition, as well as helpful discussions with Joshua Jacobs, 
Jeremy Manning, Katherine McEldoon, Sean Polyn, and Per Sederberg that benefited the article. This work was supported by a postdoctoral fellowship to C.T.W. from the German Academic Exchange Service (DAAD) and by NIMH Grants 55678 and 61975 to M.J.K. Correspondence should be sent to C. T. Weidemann, Department of Psychology, University of Pennsylvania, 3401 Walnut St., Room 302c, Philadelphia, PA 19104 (e-mail: ctw@cogsci.info).

\section{REFERENCES}

Caplan, J. B., Madsen, J. R., Schulze-Bonhage, A., AschenbrennerScheibe, R., Newman, E. L., \& Kahana, M. J. (2003). Human $\theta$ oscillations related to sensorimotor integration and spatial learning. Journal of Neuroscience, 23, 4726-4736.

Chun, M. M. (1997). Types and tokens in visual processing: A double dissociation between the attentional blink and repetition blindness. Journal of Experimental Psychology: Human Perception \& Performance, 23, 738-755.

CurRan, T. (2004). Effects of attention and confidence on the hypothesized ERP correlates of recollection and familiarity. Neuropsychologia, 42, 1088-1106.

Curran, T., DeBuse, C., \& Leynes, P. A. (2007). Conflict and criterion setting in recognition memory. Journal of Experimental Psychology: Learning, Memory, \& Cognition, 33, 2-17.

Curran, T., DeBuse, C., Woroch, B., \& Hirshman, E. (2006). Combined pharmacological and electrophysiological dissociation of familiarity and recollection. Journal of Neuroscience, 26, 1979-1985.

Curran, T., \& Friedman, W. J. (2004). ERP old/new effects at different retention intervals in recency discrimination tasks. Cognitive Brain Research, 18, 107-120.

Curran, T., \& Hancock, J. (2007). The FN400 indexes familiaritybased recognition of faces. NeuroImage, 36, 464-471.

DIEN, J. (1998). Issues in the application of the average reference: Review, critiques, and recommendations. Behavior Research Methods, Instruments, \& Computers, 30, 34-43.

Ekstrom, A. D., Caplan, J. B., Ho, E., Shattuck, K., Fried, I., \& KaHANA, M. J. (2005). Human hippocampal theta activity during virtual navigation. Hippocampus, 15, 881-889.

Hok, V., Save, E., Lenck-SAntini, P. P., \& Poucet, B. (2005). Coding for spatial goals in the prelimbic/infralimbic area of the rat frontal cortex. Proceedings of the National Academy of Sciences, 102, 46024607.

Huber, D. E., Shiffrin, R. M., Lyle, K. B., \& Ruys, K. I. (2001). Perception and preference in short-term word priming. Psychological Review, 108, 149-182.

Hwang, G., Jacobs, J., Geller, A., Danker, J., Sekuler, R., \& KaHANA, M. J. (2005). EEG correlates of verbal and nonverbal working memory. Behavioral \& Brain Functions, 1, 20.

iaria, G., Fox, C. J., Chen, J.-K., Petrides, M., \& Barton, J. J. S. (2008). Detection of unexpected events during spatial navigation in humans: Bottom-up attentional system and neural mechanisms. European Journal of Neuroscience, 27, 1017-1025.

Jacobs, J., Hwang, G., Curran, T., \& Kahana, M. J. (2006). EEG oscillations and recognition memory: Theta correlates of memory retrieval and decision making. NeuroImage, 32, 978-987.

Jensen, O., \& Tesche, C. D. (2002). Frontal theta activity in humans increases with memory load in a working memory task. European Journal of Neuroscience, 15, 1395-1399.

KAHANA, M. J. (2006). The cognitive correlates of human brain oscillations. Journal of Neuroscience, 26, 1669-1672.

KANWISHER, N. G. (1987). Repetition blindness: Type recognition without token individuation. Cognition, 27, 117-143.

Maris, E., \& Oostenveld, R. (2007). Nonparametric statistical testing of EEG- and MEG-data. Journal of Neuroscience Methods, 164, 177-190.

Meyer, D. E., \& Schvaneveldt, R. W. (1971). Facilitation in recognizing pairs of words: Evidence of a dependence between retrieval operations. Journal of Experimental Psychology, 90, 227-234

Meyer, D. E., \& Schvaneveldt, R. W. (1976). Meaning, memory structure, and mental processes. Science, 192, 27-33.

Newman, E. L., Caplan, J. B., Kirschen, M. P., Korolev, I. O.,
Sekuler, R., \& Kahana, M. J. (2007). Learning your way around town: How virtual taxicab drivers learn to use both layout and landmark information. Cognition, 104, 231-253.

Nichols, T. E., \& Holmes, A. P. (2002). Nonparametric permutation tests for functional neuroimaging: A primer with examples. Human Brain Mapping, 15, 1-25.

Poucet, B., Lenck-Santini, P. P., Hok, V., Save, E., Banquet, J. P., Gaussier, P., \& Muller, R. U. (2004). Spatial navigation and hippocampal place cell firing: The problem of goal encoding. Reviews in the Neurosciences, 15, 89-107.

Raymond, J. E., Shapiro, K. L., \& Arnell, K. M. (1992). Temporary suppression of visual processing in an RSVP task: An attentional blink? Journal of Experimental Psychology: Human Perception \& Performance, 18, 849-860.

Sauseng, P., Hoppe, J., Klimesch, W., Gerloff, C., \& Hummel, F. C. (2007). Dissociation of sustained attention from central executive functions: Local activity and interregional connectivity in the theta range. European Journal of Neuroscience, 25, 587-593.

Sederberg, P. B., Gauthier, L. V., Terushinin, V., Miller, J. F., BarNATHAN, J. A., \& Kahana, M. J. (2006). Oscillatory correlates of the primacy effect in episodic memory. Neurolmage, 32, 1422-1431.

Shelton, A. L., \& Gabrieli, J. D. E. (2002). Neural correlates of encoding space from route and survey perspectives. Journal of Neuroscience, 22, 2711-2717.

Tallon-Baudry, C., Bertrand, O., Delpuech, C., \& Permier, J. (1997). Oscillatory gamma-band $(30-70 \mathrm{~Hz})$ activity induced by a visual search task in humans. Journal of Neuroscience, 17, 722-734.

Vogel, E. K., LuCK, S. J., \& Shapiro, K. L. (1998). Electrophysiological evidence for a postperceptual locus of suppression during the attentional blink. Journal of Experimental Psychology: Human Perception \& Performance, 24, 1656-1674.

Vogels, R., SARY, G., Dupont, P., \& Orban, G. A. (2002). Human brain regions involved in visual categorization. NeuroImage, 16, 401-414.

Weidemann, C. T., Huber, D. E., \& Shiffrin, R. M. (2005). Confusion and compensation in visual perception: Effects of spatiotemporal proximity and selective attention. Journal of Experimental Psychology: Human Perception \& Performance, 31, 40-61.

Weidemann, C. T., Huber, D. E., \& Shiffrin, R. M. (2008). Prime diagnosticity in short-term repetition priming: Is primed evidence discounted, even when it reliably indicates the correct answer? Journal of Experimental Psychology: Learning, Memory, \& Cognition, 34, 257-281.

Wolfe, J. M. (1998). Visual search. In H. Pashler (Ed.), Attention (pp. 13-73). Hove, U.K.: Psychology Press.

Yuval-Greenberg, S., Tomer, O., Keren, A. S., Nelken, I., \& Deouell, L. Y. (2008). Transient induced gamma-band response in EEG as a manifestation of miniature saccades. Neuron, 58, 429-441.

\section{NOTES}

1. This particular design was inspired by the experiments of Newman et al. (2007) before we decided to conduct the analyses presented here.

2. Even though we did not separately measure familiarity with the stores, we take the fact that navigation performance was high (see the Results section) to indicate that participants were highly familiar with the stores. Also note that the total set of different stores was relatively small (only five per town). To the extent that participants were not familiar with a particular store, this should have affected the target, nontarget, and neutral store views equally, because every store served in each of these roles.

3. Navigation required only slight movements of the left thumb to press down different sides of a round pad on the controller. Speed could vary with the amount of pressure that was applied on the pad, but participants tended to navigate at full speed.

4. In order not to interfere with the interactive and dynamic task, participants received no special instructions to avoid eyeblinks or eye movements. Because of the $\pm 75-\mu \mathrm{V}$ threshold on scalp electrodes, not all of the events contributed data for every electrode.

(Manuscript received May 13, 2008; revision accepted for publication November 21, 2008.) 